\title{
Development of a near-field sensor to study the effect of glucose concentration
}

\author{
Kseniya Zavyalova ${ }^{1}$, Andrey Zapasnoy ${ }^{1}$, Aleksandr Gorst ${ }^{1}$, Aleksandr Mironchev ${ }^{1}$, Andrey Klokov ${ }^{1}$ \\ ${ }^{1}$ Tomsk State University \\ 36 Lenin Ave., Tomsk, Russia \\ zkv@mail.tsu.ru
}

\section{Extended Abstract}

In this study a near-field sensor design has been developed to study the effect of glucose concentration in a biological medium. The sensor is a combined emitter in the form of a symmetric dipole and an annular frame. The sensor is powered at the input of a symmetric dipole. This sensor was designed to maximize the amount of bound energy in its near zone. The near field of radiowave radiation should not experience absorption in the conducting medium and significant distortion due to small inhomogeneities because of its nature. Therefore, we studied the possibility of creating a sensor sensitive to changes in glucose concentration based on the near-field effect. A biological medium, such as a person's hand or wrist, is generally a conductive medium. The developed combined sensor showed its consistency in the study of solutions with different glucose contents in a numerical model.

The research was based on experimental and theoretical studies, as well as on numerical modeling of the influence of the dielectric constant of biological materials (media) on the reflected signal of the sensor.

To begin with, we modeled the sensor and tested its sensitivity on the blood layer with different values of glucose concentrations. Next, we carried out a detailed numerical study of the influence of all layers.

The main components of the biological medium are blood, fat, muscles and bones. From the point of view of diagnosing glucose in the blood of a person, the most simple and convenient places for diagnosis are his limbs (arms and legs), in particular the wrist.

In these limbs, the following layers can be conditionally distinguished: skin, which in turn is divided into epidermis and dermis (the main component of this layer); hypodermis, which forms the cell space, which includes fatty deposits and blood vessels; the next biological layer is the muscles that occupy most of the space of a human limb; in the center of all layers is a bone.

Microwave diagnostics is based on establishing the relationship of changes in the dielectric constant of the medium and the parameters of the probing signal. In theoretical studies, the dielectric constant is usually approximated using the Debye model, or a slightly modified version of it - the Cole-Cole model. It was the latter model that made it possible to calculate the permittivity of the substances listed above in a wide frequency range.

We independently calculated the dependences of the real part of the dielectric constant of blood, fat, muscle and bone on a frequency in the range from $10 \mathrm{MHz}$ to $10 \mathrm{GHz}$. As one would expect, substances containing an aqueous solution (blood, muscles and skin) have a higher dielectric constant in a wide frequency band. The same feature is also characteristic of the imaginary part of the dielectric constant.

The features of the near-field interaction of the sensor with various substances of the biological medium were studied by us based on the analysis of the behavior of the real part of the radiation power flux density (Poynting vector). The formation of such a flow is a characteristic feature of the interference interaction of overlapping evanescent fields, regardless of their origin (in this case, the opposing fields of the probe and the field reflected from the substance overlap). The calculation results allowed us to estimate the depth of radiation penetration into the studied sample of the substance.

Another important parameter of the sensor is its coordination with the studied sample of biological substance, which means that all radiated energy must penetrate into the sample. Such a parameter is the voltage standing wave coefficient, and the resistance of the near-field source should be consistent with the resistance of human blood, since the change in its dielectric constant is the most important for our consideration. During the simulation, the thickness of the layer of blood 
adjacent to the sensor was considered to be $35 \mathrm{~mm}$ (based on the diameter of the sensor and the total signal attenuation in the sample of biological substance).

As a result of numerical simulation, it was possible to construct the dependence of the reflected signal (parameter S11) on the frequency for a model of a layered medium at various concentrations of glucose in the blood. It was found that various concentrations of glucose in the blood are distinguishable in amplitude in the frequency range 3.5-7.5 $\mathrm{GHz}$, and the maximum change in amplitude is observed at a frequency of $3.5 \mathrm{GHz}$. Such a frequency shift is associated with the presence of additional (in addition to blood) layers of biological substances. After subtracting the signal, all concentrations are distinguishable in the same range, and the maximum difference is observed at a frequency of $4 \mathrm{GHz}$. The values of the amplitude of the reflected signal for different concentrations of glucose in the blood are respectively equal: at $4 \mathrm{mmol} / 1-0.8748$; at $5.3 \mathrm{mmol} / \mathrm{L}-0.64009 ; 7.5 \mathrm{mmol} / \mathrm{L}-0.47739$.

From the results of numerical simulation it follows that when the sensor is located in close proximity to the surface of the studied biological medium, signal changes caused by changes in the electromagnetic parameters of blood, skin, and muscles can be recorded. Since a change in blood glucose leads to changes in dielectric constant, the largest changes in signal level should be expected in the radiation passing through the blood vessels. A further task for study is the refinement of the model of a plane-layered medium. In particular, supplementation with venous blood with capillary blood present and isolation of the first. An important point will be the breakdown of the skin into its constituent elements, namely: dermis, epidermis, stratum corneum of the epidermis. It is also planned to investigate various variations of the parameters of biological media to determine the effect of each on the near field of the sensor.

As test samples for real experiments, we used sodium chloride solutions with different dextrose concentrations in them. The experiment was carried out using the Agilent Technologies PNA-L Network Analyzer (N5230C) vector network analyzer in a wide frequency range $(10 \mathrm{MHz}-10 \mathrm{GHz})$ for detailed analysis and selection of the frequency range with the best response. The developed sensor was leaning against the container with test samples. The container was made by $3 \mathrm{D}$ printing from ABS plastic, while at the bottom there was a hole with an attached nylon tube for draining solutions, which ensured the immobility of the installation and, as a result, reduced measurement error. The container was filled with the same amount of solution $(300 \mathrm{ml})$. The received signal for the studied samples of solutions $(1,3,4,5,7,9,10 \mathrm{mmol} / \mathrm{L})$ was normalized to the solution without dextrose $(0 \mathrm{mmol} / \mathrm{L})$. As a result, the amplitudes and phases of the normalized reflection coefficient from solutions were built and analyzed. Subtraction of the zero glucose concentration $(0 \mathrm{mmol} / \mathrm{L})$ from the obtained data shows the difference in the reflected signals, as

well as the difference at different concentrations. The results obtained are of great interest for further study and creation on this basis of an effective technology for non-invasive glucometry.

\section{Acknowledgements}

The reported study was funded by RSF project number 18-75-10101. 